HUTP-95/A045, LBL-37996, UCB-PTH-95/41

hep-th/9511164

\title{
Two-Dimensional Black Hole and Singularities of CY Manifolds
}

\author{
Hirosi Ooguri \\ Department of Physics, University of California at Berkeley \\ 366 Le Conte Hall, Berkeley, CA 94720-7300 \\ and \\ Theoretical Physics Group, Mail Stop 50A-3115 \\ Lawrence Berkeley Laboratory, Berkeley, California 94720 \\ and \\ Cumrun Vafa \\ Lyman Laboratory of Physics \\ Harvard University \\ Cambridge, MA 02138
}

We study the degenerating limits of superconformal theories for compactifications on singular $K 3$ and Calabi-Yau threefolds. We find that in both cases the degeneration involves creating an Euclidean two-dimensional black hole coupled weakly to the rest of the system. Moreover we find that the conformal theory of $A_{n}$ singularities of $K 3$ are the same as that of the symmetric fivebrane. We also find intriguing connections between $A D E(1, n)$ non-critical strings and singular limits of superconformal theories on the corresponding ALE space.

November 1995 


\section{Disclaimer}

This document was prepared as an account of work sponsored by the United States Government. While this document is believed to contain correct information, neither the United States Government nor any agency thereof, nor The Regents of the University of California, nor any of their employees, makes any warranty, express or implied, or assumes any legal liability or responsibility for the accuracy, completeness, or usefulness of any information, apparatus, product, or process disclosed, or represents that its use would not infringe privately owned rights. Reference herein to any specific commercial products process, or service by its trade name, trademark, manufacturer, or otherwise, does not necessarily constitute or imply its endorsement, recommendation, or favoring by the United States Government or any agency thereof, or The Regents of the University of California. The views and opinions of authors expressed herein do not necessarily state or reflect those of the United States Government or any agency thereof, or The Regents of the University of California.

Lawrence Berkeley Laboratory is an equal opportunity employer. 


\section{Introduction}

One of the central issues in the recent progress in understanding non-perturbative aspects of string theory has been the realization that singular conformal theories which correspond to singular points in moduli of string compactifications in many cases signal the existence of nearly massless solitons [1], [2], [3], [4]. Since these nearly massless modes are left out of the low energy spectrum of elementary excitations of strings, integrating them out will lead to a singular low energy description. As examples of this phenomenon one can mention the enhanced gauge symmetry of type IIA once we compactify on singular limits of $K 3$ (or ALE spaces) where the gauge group is $A D E$ depending on the singularity type [2] and the Dynkin diagram is determined by the intersection form of vanishing 2-cycles. Another example is provided by the compactifications of type IIB strings on singular Calabi-Yau threefolds. If we have a conifold singularity, where a 3-cycle vanishes, one expects a massless solitonic hypermultiplet (carrying Ramond-Ramond charge) [3]. It is therefore important to understand the nature of the singularity of the conformal theory in these classes of examples. This is the main aim of this paper. As a byproduct, the results presented here have led to a dual perturbative string description of the massless solitons in both of these classes [5], where the nearly massless soliton is realized as an elementary state of the D-string.

The organization of this paper is as follows. We will first discuss the case of CalabiYau 3-fold in the presence of conifold singularity. Then we consider ALE spaces with ADE singularities and present a conformal theory description of them. Rather surprisingly we find that they are equivalent to symmetric fivebrane solutions. We will establish this at the level of conformal theories and give a geometric explanation of this phenomenon based

on 'stringy cosmic strings' [6]. Also some mysterious connections are found with the $(1, n)$ conformal theories coupled to gravity, following a similar connection between the $c=1$ at the self-dual radius and the conifold singularity of Calabi-Yau threefold [7]. In particular just as the conifold singularity is topologically captured by the $N=2$ topological theory, one expects that the $A D E$ singularities on ALE are captured by $N=4$ topological theory [8]. We find indications that this latter $N=4$ topological system is related and possibly equivalent to $(1, n)$ conformal system coupled to the two-dimensional gravity. 


\section{Conifold in Three Dimensions and Massless Soliton}

We can gain information on the spectrum of the conformal field theory by computing [9]

$$
F_{1}=\frac{1}{2} \int_{\mathcal{M}_{1}} \frac{d^{2} \tau}{\operatorname{Im} \tau} \operatorname{tr}^{\prime}(-1)^{F_{L}+F_{R}} F_{L} F_{R} q^{H_{L}} \bar{q}^{H_{R}},
$$

where $\operatorname{tr}^{\prime}$ means a trace over the Hilbert space of the $N=2$ superconformal non-linear sigma-model on the Calabi-Yau threefold in the Ramond sector with zero-energy (chiral) states eliminated, and $F_{L}$ and $F_{R}$ are left and right-moving fermion number operators. This is a standard expression for one-loop partition function of a topological string on the CalabiYau manifold, but it can also be interpreted as computing one-loop correction to $R^{2}$ terms in the low energy effective action of the type II superstring theory [10], [11]. In general, $F_{1}$ computed for a compact Calabi-Yau manifold is finite since the spectrum of the sigmamodel should be discrete with a finite mass gap and therefore $\operatorname{tr}^{\prime}(-1)^{F_{L}+F_{R}} F_{L} F_{R} q^{H_{L}} \bar{q}^{H_{R}}$ becomes exponentially small as $\tau \rightarrow \infty$. In [9], $F_{1}$ is computed in four different families Calabi-Yau manifolds, each of which has a conifold singularity in the moduli space. In the neighborhood of the conifold, local structure near the singularity of the Calabi-Yau manifolds can be described in the universal form as the quadric [12

$$
y_{1}^{2}+y_{2}^{2}+y_{3}^{2}+y_{4}^{2}=\mu, \quad\left(y \in \mathbf{C}^{4}\right) .
$$

As $\mu \rightarrow 0$, the quadric develops a singularity with a collapsing 3-cycle. It turned out that in all the four cases, $F_{1}$ behaves as 1

$$
F_{1} \sim-\frac{1}{12} \log |\mu|^{2}
$$

as $\mu \rightarrow 0$. This was further confirmed by computation of $F_{1}$ in many other examples studied in [13]. To be consistent with Strominger's interpretation, this logarithmic divergence should be due to the virtual nearly massless solitonic loop [14]. On the other hand, from the point of view of conformal field theory, this divergence signals vanishing of mass gap in the spectrum since contributions from massive excitations to $F_{1}$ are finite. This implies that the perturbative string theory acquires additional light particles in the neighborhood of the conifold. These light particles are unstable in string perturbation theory.

1 At genus-one, the topological string actually computes $\partial_{\mu} F_{1}$ since we need to fix one-point on a torus. More generally for $F_{g}$ we would be interested in $F_{g \mid \bar{\mu} \rightarrow 0}$ which is the topological expansion of $F_{g}$ in the conifold limit. 
Hints that the topological theory of Calabi-Yau threefold are related to $c=1$ strings at self-dual radius first appeared in [15] where it was shown to be described by a $\hat{c}=3$ twisted Kazama-Suzuki model [16] for a coset $S L(2) / U(1)$ at level $k=3$. In fact this explained the observation in [17] that the topological theory of this model at genus- $g$ gives Euler characteristic of moduli space of Riemann surfaces which is the same as the partition function of $c=1$ string at the self-dual radius [18], [19]. The equivalent Landau-Ginzburg description for it is given by a superpotential $W=-\mu x^{-1}$ by "analytic continuation" from the $S U(2) / U(1)$ Kazama-Suzuki model where a superpotential is $W=x^{k+2}$ for level $k$ [20], [21]. More evidence in favor of this description was found by studying the correlators of this model in the Landau-Ginzburg framework and obtaining the $c=1$ tachyon amplitudes 22], 23].

Further evidence for the $c=1$ - Calabi-Yau connection emerged from [10] where it was noticed that as a function of $\mu$ the genus- $g$ partition function of topological theory near conifold limit scales as $F_{g} \sim \mu^{2-2 g}$, which is the same as one expects for $c=1$ theory coupled to gravity. This connection was explained in [7] by noting that the sigma-model for (2.1) is related to the Landau-Ginzburg model with a superpotential

$$
W(x, y)=-\mu x^{-1}+y_{1}^{2}+y_{2}^{2}+y_{3}^{2}+y_{4}^{2}
$$

The equation $W=0$ in a weighted projective space $W C P_{-2,1,1,1,1}^{4}$ satisfies the CalabiYau condition with the central charge $\hat{c}=3$ [24]. Note that adding quadratic fields does not change the conformal fixed point and is a standard technique for relating Calabi-Yau compactifications with Landau-Ginzburg theories [25]. When $x \neq 0$, we can set $x=1$ without any loss of generality and $W=0$ in this patch is equivalent to the deformed conifold (2.1). As far as $\mu \neq 0$, the patch with $x=0$ is associated with $\partial W=\infty$ and would not arise in the geometric description of the theory (at least in a suitable regime of field space - it would be interesting to check these from the viewpoint of linear sigma models [26]). These results in particular explain the universal behavior of $F_{1}$ near the conifold singularity. Moreover for $g>1$, using the result for $c=1$ string partition function we learn that $F_{g}$ of the Calabi-Yau near the conifold limit is given by

$$
F_{g}=\frac{B_{2 g}}{2 g(2 g-2)} \mu^{2-2 g}
$$

where $B_{n}$ is the Bernoulli number and $B_{2 g} / 2 g(2 g-2)$ is the Euler class of the moduli space of genus- $g$ Riemann surfaces. At $g=2$, this expression is consistent with the conifold limit 
of the result in [10] where the $g=2$ topological string amplitude is computed for the quintic threefold. More recently it was shown that the behavior (2.3) is consistent to all order in $g$ with the corresponding correction to $R^{2} F^{2 g-2}$ in four dimensions where one considers a virtual loop of a nearly massless soliton [27] thus generalizing the work in [14] to all loops and providing a strong check for the conjecture of Strominger that a nearly massless solitonic object 'explains' topological singularity of conformal theory in the conifold limit.

\subsection{Geometrical Picture of the Conformal Theory}

The conformal theory describing the conifold is thus the untwisted version of the $S L(2) / U(1)$ Kazama-Suzuki model with $k=3$. The non-supersymmetric version of this is the Euclidean black hole system in two dimensions [28]. The geometrical picture developed there is as follows (see also [29]): The geometry is that of an infinitely long "cigar." For most of the geometry, if we are far enough from the tip of the cigar, we have a cylindrical geometry parameterized by $X$ running over a circle and $\phi$ along the length of cigar. Moreover there is a linear dilaton in the $\phi$ direction with the string coupling becoming weaker as we go along the cigar. The supersymmetric description has basically the same bosonic piece, but in addition the standard fermionic partners. As for the physical states of the $c=1$ string at the self-dual radius, they come from the discrete representations of $S L(2)$ [15 and they can be viewed as bound states at zero energy near the tip of the cigar [29]. On the other hand the waves propagating down the cigar correspond to non-topological states, and are BRST trivial in the sense of the $c=1$ string - However those are precisely the ones which are responsible for the continuous spectrum of the Calabi-Yau near the conifold. This interpretation is consistent with the considerations of unitarity: We expect that the conformal theory of Calabi-Yau has a unitary spectrum. The analysis of [30] concluded that for this level of $k$ essentially none of the discrete representations of the coset model is unitary (the exception being the one corresponding to the cosmological constant operator which is also a real deformation of Calabi-Yau), whereas all the continuous representations are unitary. In fact this meshes nicely with the picture advocated in [31] if we think of the length of the tube as being related to $-\log \mu$ and connected to the rest of the Calabi-Yau conformal theory. The states of $c=1$ string which are bound to the tip of the cigar do not communicate with the rest of the Calabi-Yau because the coupling is weaker by a factor of $\mu$, and moreover they do not arise as physical states of the Calabi-Yau compactification, consistent with unitarity requirements. 
The picture advocated in [31] was in connection with the explanation of the unreliability of string perturbation theory. In such a picture the coupling being infinitely strong at the tip of the cigar in the limit $\mu \rightarrow 0$ shows that the perturbation theory should break down if $\mu<\lambda$, where $\lambda$ is the string coupling constant. In fact this already follows from the computation of the topological amplitude above: Namely if we turn on a background $F$ and compute the correction to $R^{2}$, as explained above, near the conifold the genus- $g$ correction goes as $F_{g} \sim \mu^{2-2 g} \lambda^{2 g-2}$ and thus if $\mu<\lambda$ each successive correction gets larger and the perturbation sum becomes unreliable? 2 .

The main puzzle is how does this fit with the geometry of the Calabi-Yau near the conifold? To begin with we have a vanishing $S^{3}$. Where did the cigar shaped object appear? When did we turn on the dilaton? We will be able to shed some light on this after our discussion on the singularities of the conformal theory near $K 3$ degenerations.

\section{3. $A D E$ Singularities in $K 3$ and Fivebrane Solution}

Let us turn our attention to singularities in $K 3$ surfaces. From the duality between type II and heterotic string models in six dimensions, it is expected that the perturbative type II string on $K 3 \times \mathbf{R}^{6}$ will have singularities when the dual heterotic string on $T^{4} \times \mathbf{R}^{6}$ approaches enhanced gauge symmetry points [2]. These special points for $K 3$ are modeled (and generalized) by the ALE spaces which have $A D E$-type singularities given by

$$
\begin{aligned}
A_{n-1}: & y_{1}^{n}+y_{2}^{2}+y_{3}^{2}=\mu \quad(n \geq 2) \\
D_{\frac{n}{2}+1}: & y_{1}^{\frac{n}{2}}+y_{1} y_{2}^{2}+y_{3}^{2}=\mu \quad(n: \text { even } \geq 6) \\
E_{6}: & y_{1}^{4}+y_{2}^{3}+y_{3}^{2}=\mu \\
E_{7}: & y_{1}^{3} y_{2}+y_{2}^{3}+y_{3}^{2}=\mu \\
E_{8}: & y_{1}^{5}+y_{2}^{3}+y_{3}^{2}=\mu, \quad\left(x \in \mathbf{C}^{3}\right)
\end{aligned}
$$

with appropriately chosen values of the Kähler moduli [33]. As in the case of Calabi-Yau threefolds, we can add one more variable $x$ and consider corresponding Landau-Ginzburg

2 This story is very similar and possibly related to the issue of heterotic/type I duality recently discussed in [32]. 
models with superpotentials

$$
\begin{aligned}
A_{n-1}: & W=-\mu x^{-n}+y_{1}^{n}+y_{2}^{2}+y_{3}^{2} \\
D_{\frac{n}{2}+1}: & W=-\mu x^{-n}+y_{1}^{\frac{n}{2}}+y_{1} y_{2}^{2}+y_{3}^{2} \\
E_{6}: & W=-\mu x^{-12}+y_{1}^{4}+y_{2}^{3}+y_{3}^{2} \\
E_{7}: & W=-\mu x^{-18}+y_{1}^{3} y_{2}+y_{2}^{3}+y_{3}^{2} \\
E_{8}: & W=-\mu x^{-30}+y_{1}^{5}+y_{2}^{3}+y_{3}^{2} .
\end{aligned}
$$

It is straightforward to verify that the equation $W=0$ satisfies the Calabi-Yau condition with $\hat{c}=2$. Note also that, as is usual in Gepner models, to get a connection with CalabiYau, we have to mod out by $\exp \left(2 \pi i J_{0}\right)$ which imposes the charge integrality condition and summing over the spectral flow [34]. For example for the $A_{n-1}$ series this results in modding out by a $Z_{n}$.

Let us analyse the Hilbert space structure of these models. The $y$-dependent parts of (3.2) are nothing but the superpotentials of the $N=2$ superconformal minimal models associated to the $A D E$-type modular invariants [20], [21] with

$$
\begin{aligned}
\hat{c}=1-\frac{2}{n} \quad\left(\text { for } A_{n-1} \text { and } D_{\frac{n}{2}+1}\right. & \\
& \left.n=12,18,30 \text { for } E_{6}, E_{7}, E_{8}\right) .
\end{aligned}
$$

On the other hand, the Laudau-Ginzburg model with a potential $x^{-n}$ is equivalent to the Kazama-Suzuki model for the coset $S L(2, R)_{n+2} / U(1)$ at the critical point. Above we presented evidence for this analytic continuation in the form of the superpotential $W$ from $S U(2)$ to $S L(2)$ when $n=1$ and we are assuming this is generally valid. Therefore the Landau-Ginzburg models given by the potentials (3.2) are products of the Kazama-Suzuki model and the $N=2$ minimal models modded out by an appropriate discrete group:

$$
\frac{\frac{S L(2)}{U(1)} \times \frac{S U(2)}{U(1)}}{G}
$$

where $G=Z_{n}$ in the $A_{n-1}$ case. Before going to the detail of the conformal theory construction and in particular the orbifoldizing of (3.3) by $G$, we would like to mention what the outcome is. Let us first consider the $A_{n-1}$ series. The bosonic piece of $S L(2) / U(1)$ coset model can be viewed geometrically as a cigar shaped object [28] which is physically the Euclidean two-dimensional black hole. This is a non-compact space, and except for a finite region near the tip of the cigar it can be viewed as a semi-infinite cylinder. We 
will coordinatize the length of it by $\phi$ and the circle coordinate we will denote by $\tilde{X}$ with radius $R=\sqrt{2 n}$ (which can be read off from the level of the affine Lie algebra). Moreover, there is a linear dilaton for $\phi$, i.e. it is a Feigin-Fuchs system (this is true if we are far enough from the tip of the cigar). In addition we have, in this region, two free fermionic partners of $\phi$ and $\tilde{X}$. As for the supercoset $S U(2) / U(1)$, it is more difficult to describe geometrically, but basically they are the representation of the parafermionic algebra together with a free boson (whose momentum is correlated with representations of the parafermionic system). In modding out by $G=Z_{n}$, the $Z_{n}$ acts trivially on the fermionic pieces of $S L(2) / U(1)$ coset model, but acts on the momentum (winding) states around $\tilde{X}$ by $n$-th roots of unity. At the same time, it also acts on the free bosonic part of the $S U(2) / U(1)$ minimal model. The net effect of this modding is to recombine $\tilde{X}$ and the free boson in the minimal model into two bosons of radii $\sqrt{2(n-2)}$ and 1 . The momentum of the boson with radius $\sqrt{2(n-2)}$, which we denote by $H$, is still correlated to the representations of the parafermion algebra. On the other hand, the boson at $R=1$ is decoupled and can be converted into two free fermions. The parafermionic system together with the $H$ field forms a bosonic $S U(2)$ system. Thus we finally end up after orbifoldization by $G$ with a bosonic system consisting of $S U(2)$ at level $k=n-2$ and a Feigin Fuchs system $\phi$ with background charge. We have also 4 free fermions, three of which make an $N=1$ superconformal $S U(2)$ model and the last one is a superpartner of $\phi$. The background charge of $\phi$ is tuned so that the total $\hat{c}$ equals 2 . This is the same as the symmetric fivebrane conformal theory [35] with a charge associate to the $B$ field ( $H$ charge) equal to $n$. Note that our system is a capped version of [35], in the sense that instead of the $\phi$ going off to infinity, where the coupling becomes infinitely strong, we end up instead at the tip of the cigar. This is the region where the splitting of the $S L(2) / U(1)$ coset system to $\phi, \tilde{X}$ fails.

Now we come to a more detailed description of the orbifoldization above using the characters of conformal field theory, concentrating on how the orbifoldization by $G$ leads to the $S U(2)$ characters. To perform the orbifoldization (3.3), we first note that the $U(1)$ current $J$ of the $N=2$ algebra in the Kazama-Suzuki model $S L(2, R)_{n+2} / U(1)$ takes the form

$$
J=\psi \bar{\psi}+i \sqrt{\frac{2}{n}} \partial X
$$

where $\psi, \bar{\psi}$ are free fermions that are superpartners of the bosonic coset $S L(2, R) / U(1)$ and $X$ is a free scalar living on $S^{1}$ with radius $R=\sqrt{2 / n}$ (where $X$ is dual to $\tilde{X}$ described 
above). This can be shown in the following way. In the Kazama-Suzuki model, the $N=2$ superconformal algebra are realized as

$$
\begin{aligned}
G^{+} & =\psi J^{+}, \quad G^{-}=\bar{\psi} J^{-} \\
J & =\left(1+\frac{2}{n}\right) \psi \bar{\psi}-\frac{2}{n} J^{3}
\end{aligned}
$$

where $J^{ \pm}, J^{3}$ are the generators of $S L(2, R)_{n+2}$. To take the quotient by $U(1)$, we introduce an $U(1)$ gauge field $A=i \partial X, \operatorname{BRST}$ ghosts $(B, C)$ of weights $(1,0)$ and a BRST current

$$
J_{B R S T}=C\left(\psi \bar{\psi}-J^{3}-i \sqrt{\frac{n}{2}} \partial X\right) .
$$

The coefficient in front of $X$ is determined by requiring the nilpotency of the BRST charge $Q_{B R S T}$. This also implies that $X$ should live on $S^{1}$ of radius $R=\sqrt{2 / n}$. It follows that the $U(1)$ current $J$ in (3.5) is equal to (3.4) up to a BRST trivial operator.

Now we are ready to perform the orbifoldization of the Landau-Ginzburg model, noting that the generator of $G$ acting on the $S L(2) / U(1)$ piece can be identified with $\exp \left(2 \pi i J_{0}\right)$ and so in particular using (3.4) it acts only on the $X$ piece of the coset. The relevant partition function of the $X$ system is given by

$$
\frac{\vartheta_{m, n}(\tau)}{\eta(\tau)}
$$

where

$$
\vartheta_{m, n}(\tau)=\sum_{s \in \mathbf{Z}} q^{n\left(s+\frac{m}{2 n}\right)^{2}}
$$

For the $S U(2) / U(1)$ system, which is the $N=2$ minimal model, we use a character of an irreducible representation of the $N=2$ superconformal algebra, which is denoted by $c h_{l, m^{\prime}}^{(n-2)}(\tau)$, where $l$ and $m^{\prime}$ are related to the conformal weight $h_{l, m^{\prime}}$ and the $U(1)$ charge of the highest weight state $Q_{m^{\prime}}$ as

$$
h_{l, m^{\prime}}=\frac{l(l+2)-m^{\prime 2}}{4 n}, \quad Q_{m^{\prime}}=\frac{m^{\prime}}{n} .
$$

The charge integrality condition, which is imposed by the orbifoldization by $G$, requires that we choose $m$ in (3.6) to be equal to $m^{\prime}$ in $c h_{l, m^{\prime}}^{(n-2)}$ so we only consider the combination

$$
\frac{\vartheta_{m, n}(\tau)}{\eta(\tau)} \cdot c h_{l, m}^{(n-2)}(\tau)
$$


To sum over the spectral flow, we make use of the following expression for the $N=2$ character [36]

$$
c h_{l, m}^{(n-2)}(\tau)=\sum_{m^{\prime}=-n-1}^{n-2} c_{l, m^{\prime}}^{(n-2)}(\tau) \vartheta_{m^{\prime} n-m(n-2), n(n-2)}(\tau / 2),
$$

where $c_{l, m^{\prime}}^{(n-2)}(\tau)$ is the string function (= parafermionic partition function $\times \eta(\tau)$ ) of the affine $S U(2)$ algebra. The string function can be obtained by expanding a character of an irreducible representation of the affine $S U(2)$ algebra at level $(n-2)$ with the highest weight $l, \chi_{l}^{(n-2)}(\tau)$, in terms of the theta-function as

$$
\chi_{l}^{(n-2)}(\tau)=\sum_{m} c_{l, m}^{(n-2)}(\tau) \vartheta_{m, n-2}(\tau)
$$

The information on the $U(1)$ charge is carried by the theta-function piece of this expression. Thus the problem reduces to computing

$$
\sum_{m} \vartheta_{m, n}(\tau) \vartheta_{m^{\prime} n-m(n-2), n(n-2)}(\tau / 2)
$$

This can be done by using the multiplication formula of the theta-function. By using (3.8), we can recombine the string function and the theta-function into the $S U(2)$ character as

$$
\begin{aligned}
& \sum_{m=-l}^{n-1-l} \frac{\vartheta_{m, n}(\tau)}{\eta(\tau)} \cdot \operatorname{ch}_{l, m}^{(n-2)}(\tau)= \\
& =\prod_{r=1}^{\infty}\left(1+q^{r-1 / 2}\right)^{2} \cdot \chi_{l}^{(n-2)}(\tau)
\end{aligned}
$$

Thus we have shown that the orbifoldization of the $N=2$ minimal model combined with one free scalar $X$ coming from the Kazama-Suzuki model gives the $S U(2)_{n-2}$ WZW for the $A$-series (where the left and right characters are identical) plus two free fermions. It is straight-forward to generalize this construction for the $D$ - and $E$-series of ALE above, with the modification that the resulting $S U(2)$ partition function will be the $D$ or $E$-type modular invariant [37. This is clear because the corresponding modular combinations for the $N=2$ minimal models follows that of the underlying $S U(2)$. Note that, generally speaking, the orbifoldization of the $\hat{c}=2$ Landau-Ginzburg model introduces the affine $S U(2)$ symmetry of level 1 , which is a part of the (small) $N=4$ superconformal algebra [38], 39], and which is different from the $S U(2)_{n-2}$ symmetry manifesting itself in (3.9). 
The remainder of the Kazama-Suzuki model, apart from the two fermions of the $S L(2) / U(1)$ model, is a free scalar $\phi$ with a background charge $Q_{\phi} \sim 1 / \sqrt{n}$. The easiest way to see it is to use the Wakimoto construction of $S L(2, R)$ which realizes $J^{ \pm}, J^{3}$ in terms of one scalar field $\phi$ and a pair of bosonic ghosts $(\beta, \gamma)$ of weights $(1,0)$. Following the analysis of [15], one finds that $(\beta, \gamma)$ and $(B, C)$ makes the Kugo-Ojima quartet and decouple (in a suitable region of field space 'away' from the tip of the cigar). The remaining $\phi$ has a background charge $Q_{\phi}$, and the screening operators of the Wakimoto construction go over to the screening operators for $\phi$.

To summarize, we have found that the orbifoldized Landau-Ginzburg model with the superpotential given by (3.2) is equivalent to the $S U(2)_{n-2}$ WZW model, four free fermions and one scalar with the background charge $Q_{\phi}$. In particular, the resulting model has $S U(2)_{n-2} \times S U(2)_{1}$ symmetry. Combined with the free scalar $\phi$ and the (small) $N=4$ supercurrent, they generate the (large) $N=4$ superconformal algebra 40.

In claiming a connection between ALE spaces and LG models one has to precisely specify the Kähler moduli in order to get a singular conformal theory [33]. For example consider the $A_{1}$ singularity. If we consider it as an orbifold $R^{4} / Z_{2}$, the usual orbifold CFT is not singular precisely because the periodic $B$ field in the orbifold model is $B=\pi$ and not zero. If we adjust $B$, keeping the sphere collapsed, there are two values for which the resulting theory has an extra $Z_{2}$ symmetry: $B=\pi, 0$. The case $B=0$ is the one which gives the singular conformal theory. In fact, in our models, the Kähler moduli are automatically tuned such that the $B$ fields are zero. To see this let us consider the $A_{1}$ case as an example. In general, the orbifoldized Laudau-Ginzburg models have discrete symmetries so called quantum symmetries, which fix the value of the the Kähler moduli [41]. For the case of $A_{1}$ considered above we have to mod out by a $Z_{2}$ so we will indeed get a $Z_{2}$ quantum symmetry in the orbifoldized model. So we are either at $B=0$ or $B=\pi$. Since the concrete description of our conformal theory given above shows that it is not equivalent to standard $R^{4} / Z_{2}$ orbifold CFT, which has $B=\pi$ we conclude that $B=0$ in the above construction.

As mentioned before for the case of the $A_{n-1}$ series, the orbifold Laudau-Ginzburg model we just constructed is identical to the conformal field theory of the symmetric fivebrane solution with the $H$ charge $n$ [42], [35], [43]. The target space geometry of the fivebrane solution has an asymptotically flat region and there is a semi-infinite cylinder (wormhole) attached to its center. The scalar field $\phi$ corresponds to the radial coordinate along the cylinder, and the $S U(2)_{n-2}$ WZW model describes the $S^{3}$ part, which is the 
cross-section of the cylinder. The volume of the cross-section is proportional to the $H$ charge $n$ [42]. Thus if this were a bosonic system, the level of the $S U(2)$ algebra would be equal to $n$. However we must also take into account four fermions which are superpartners of $\phi$ and the $S U(2)$ WZW model. Initially they are are not free but are coupled to the generalized connections on the target space. However in the fivebrane solution, these connections are parallelized by the anti-symmetric tensor field and we can transform the fermions into free ones by chiral gauge rotations. This procedure causes the shift $n \rightarrow n-2$ through chiral anomalies.

Recently Witten has examined the phase structure of the linear sigma model for the $A_{1}$ type singularity and conjectured that it should be related to the fivebrane solution based on the similarity between their global geometric structures [31]. Here we have proven his conjecture by studying the Hilbert space structure of the model explicitly, and we have determined the value of the $H$ charge to be 2 . We also extended it to all $A_{n-1}$, with the result that the ALE singularity given by $A_{n-1}$ is equivalent to the conformal theory describing the symmetric fivebrane with the charge $n$. There are two subtleties to consider: The first one is that if we consider type IIA(B) on ALE it is equivalent to type $\operatorname{IIB}(\mathrm{A})$ on the symmetric fivebrane. This exchange is easy to see, because in the LG model above we could vary the complex structure of ALE, i.e. it is a B-model description of the theory, whereas in the symmetric fivebrane, the description is in the A-model (i.e. the sigma model origin for supercharges). This statement will become even more clear in the next section which is related to resolving yet another puzzle: How come we got the $H$ field and the dilaton field turned on, while we thought we were talking about $K 3$ target space? A hyperkähler metric on $K 3$ is uncorrected in worldsheet theory and gives rise to a conformal theory. Where do we get the dilaton and $H$ fields from? This is the topic of the next section, where we use the stringy cosmic string description of $K 3$ given in [6] to show how the $H$ and dilaton arises as well as rederiving the relation between the value of charge of the symmetric fivebrane and the value of $n$ in the $A_{n-1}$ type singularity.

\section{Stringy Cosmic Strings, $K 3$ and its Dual}

Let us recall the construction of stringy cosmic string [6]. We start with compactification on a two torus $T^{2}$. The moduli for this compactification is given by two complex parameters $(\tau, \rho)$ where $\tau$ parameterizes the complex structure of $T^{2}$ and $\rho$ parameterizes 
the complexified Kähler class of $T^{2}$. Note that both are defined up to an $S L(2, \mathbf{Z})$ transformation. Moreover there is an exchange symmetry $\tau \leftrightarrow \rho$ which is obtained by applying $R \rightarrow 1 / R$ to one of the circles. Viewed in eight-dimensional terms, the moduli $\tau$ and $\rho$ appear as complex scalar fields. We consider finding solutions to the low energy eightdimensional equations of motion, where fields depend only on 2 extra parameters (i.e. we are interested in keeping a six-dimensional Poincare invariance). Let us denote the extra two-dimensional space by $z$. A nice class of such solutions [6] can be obtained by taking $\tau(z)$ to depend holomorphically on $z$, and to take $\rho$ to be constant, independent of $z$. In making sense of such a solution one has to recall that $\tau$ is defined only up to $S L(2, \mathbf{Z})$ and so we are allowed to have jumps in $\tau$ up to this transformation. Geometrically what this means is that as we go around some cycles on the $z$-plane, if we follow the compactified $T^{2}$, it comes back to itself, but with some monodromy. In other words if we follow a given cycle of $T^{2}$ continuously it does not necessarily come back to itself. Geometrically this is a perfectly nice condition. If around some point say $z=0$ we have monodromies, it must mean that the $T^{2}$ fiber above it is singular where a cycle vanishes, and the monodromy of cycles around $z=0$ can give additions of this vanishing cycles. Recall that a degenerating $T^{2}$ can be viewed near its degeneration point by

$$
x y=q
$$

where $x, y$ are coordinates on some patch, and $q$ denotes the plumbing fixture modulus. As $q \rightarrow 0$ we get a torus with a vanishing cycle. Now let us replace $q \rightarrow z$ and think of the degenerating family of elliptic curves over the $z$-plane given locally by $x y=z$. At $z=0$ we have our degenerating torus. Note however, that the total space is not singular despite the fact that the fiber is singular. The easiest way to see this is to consider $x, y$ as the coordinates. Clearly $z$ is fixed uniquely in terms of $x, y$ as $z=x y$ and so the space looks locally like $\mathbf{C}^{2}$. The points over which the fiber degenerates are the points where there is a 'cosmic string' (if we were dealing with compactifications down to 4).

We can, however, develop a singularity if we get $n>1$ singular fibers approach each other. Let $a_{i}$ denote the $z$ coordinate of $n$ singular fibers with $i=1, \ldots, n$. Suppose the singularities are such that the point on $T^{2}$ where they degenerate are the same. Then a local description of this singularity can be given as

$$
x y=\prod_{i}\left(z-a_{i}\right)
$$


and if we take $a_{i} \rightarrow 0$ we would be discussing the $A_{n-1}$ singularity at $x=y=z=0$.

In solving the eight-dimensional equations of motion, one realizes that since there is energy density due to the variation of the scalar field $\tau$ there is curvature. In fact it was shown in [6] that if we have $n$ points where the fiber degenerates in the way we discussed above the $z$-plane will have a conical asymptotic with conical angle given by $2 \pi\left(1-\frac{n}{12}\right)$. In particular with $n=12$ we get a cylindrical behavior and with $n=24$ the $z$-plane compactifies to a sphere and the total space is compact. This compact space is nothing but $K 3$ !

The low energy equations of motion that were solved to give the stringy cosmic string solution are inapplicable precisely where the fiber degenerates. However it was argued in [6] that there exists a metric on the total space which leads to a hyperKähler metric on the total space. This is also clear in the case where $n=24$ as $K 3$ admits a hyperKähler metric and we can view $K 3$ as an elliptic fibration over the sphere (i.e. $K 3$ is an elliptic surface).

It was also observed in [6] that we can exchange the role of $\tau \leftrightarrow \rho$ in the above construction, and the $T$-duality of the problem implies that we must have still a solution to the equations of motion. This of course means that type IIA in one case is equivalent to type IIB on the dual. Once we apply the duality, the metric of $T^{2}$ is now varying as well as the $B$ field, because $\rho=\frac{B}{2 \pi}+i \sqrt{G}$ on $T^{2}$. Thus the analog of the singular fibers around which we have the monodromy $\tau \rightarrow \tau+1$ now corresponds to a fixed $\tau$ with $\rho \rightarrow \rho+1$, i.e., $B \rightarrow B+2 \pi$. This in particular means that if we take a three cycle consisting of a circle $S^{1}$ around the point with degenerate fiber, times the fiber $T^{2}$ and integrate

$$
\frac{1}{2 \pi} \int_{T^{2} \times S^{1}} H=\int_{T^{2} \times S^{1}} \frac{d B}{2 \pi}=\left.\frac{1}{2 \pi} \Delta B\right|_{S^{1}}=1
$$

we have one unit of $H$ charge in that region. Note also that hidden in this discussion is that there is now also a dilaton turned on. To see this note that the duality that exchanges $\tau \leftrightarrow \rho$ also changes the dilaton so that $\rho_{2} / \lambda^{2}=\rho_{2} \exp (-2 \phi)$ is invariant. Thus in the new solution $\exp (2 \phi)=A \sqrt{G}$ for some constant $A$. Note that if we consider the case where $n$ cosmic strings come together, where we would get $A_{n-1}$ singularity, we will have in the dual $\tau \leftrightarrow \rho$ description $n$ units of $H$ charge, with the dilaton turned. This is beginning to look like the symmetric fivebrane solution of [35]. However to be more explicit we need to go beyond the adiabatic approximation used in [6] where roughly speaking the metric is split to a block diagonal form involving $T^{2} \times S^{2}$. Given that the singularity takes place 
at a given point in four space, this adiabatic approximation, which treats all points on $T^{2}$ symmetrically, misses even the symmetries of the leading singularity. Note that the singularity occurs at a given point in four space, which is of the same geometry as the symmetric fivebrane and given that it carries an $H$ charge, it is natural to expect that the leading singular piece of the two conformal theories be equivalent, as was demonstrated in the previous section.

\section{K3 and Calabi-Yau Threefold Singularities}

In section three of this paper we discussed the singularity corresponding to ALE spaces and in section two that of the conifold singularity. Is there a connection between them? The answer is yes, as anticipated in the description of conifold singularity given in 44] and used in [5]: We can consider compactifying down to six dimensions on $T^{2} \times T^{2}$ and talk about the variation of each of the complex structures $\tau_{1}$ and $\tau_{2}$ of the two $T^{2}$ as a function of an extra $z$-plane (this was also briefly considered in [6]). Now as a function of the $z$-plane the $\tau_{1}(z)$ and $\tau_{2}(z)$ might be singular at different points. Let us denote the coordinates of the degenerating first torus by 'plumbing fixture' coordinates $x, y$ as before, and those of the second one by $u, v$. Let us assume that the first torus degenerates at $z=0$ and the second one at $z=\mu$ for $\mu$ close to zero. Then locally the description of the three manifold looks as

$$
x y=z \quad u v=z-\mu
$$

which, by eliminating $z$ is equivalent to

$$
x y-u v=\mu
$$

which is the conifold singularity. We will now describe this in the conformal theory language and show how the conifold conformal theory is related to that for the ALE space. Before doing so, let us generalize the above singularity to the one corresponding to $c=1$ at radius $n$ times the self-dual value. In that case the singularity can still be given a description as above where $n$ cosmic strings of the first torus come together on the $z$-plane, i.e. we have

$$
x y=z^{n} \quad u v=z-\mu
$$

which upon eliminating the $z$ variable give the $R=n / \sqrt{2}$ singularity 445]

$$
x y=(u v+\mu)^{n}
$$


Now it is clear how to write down the conformal theory corresponding to these singularities. Consider the Landau-Ginzburg theory

$$
W=\zeta^{-n}+z^{n}+a^{2}+b^{2}+\Lambda(z-x y) .
$$

This is the same superpotential as the $A_{n-1}$ singularity except for the additional fields $\Lambda, x$ and $y$ and the interaction $\Lambda(z-x y)$. Upon integrating out $\Lambda$ we would get the identification of $z=x y$ and the superpotential becomes

$$
W=\zeta^{-n}+(x y)^{n}+a^{2}+b^{2}
$$

Note that for $n=1$ this is the same superconformal theory as we discussed in section two, for the Calabi-Yau conifold and it is known. For other values of $n$, apart from the above LG description, we do not have the full conformal theory description. Anyhow this rederivation of the conifold conformal theory shows a parallel consistency between the two classes of conformal theories we discussed and their geometric relation between the two explained above. It also explains the appearance of two-dimensional Euclidean black hole in the discussion of the conifold singularity.

This also raises the question of the conformal theory of 'one cosmic string', i.e. symmetric fivebrane with charge 1 . This is the case where the level of the $S U(2)$ theory is -1 , which presumably means that we have to interpret it as $S L(2)$ at level 1 . Thus we end up with the product of two $S L(2) / U(1)$ 's one at level 1 and the other at level 3 . So it seems that the geometry for one symmetric fivebrane has a different description from the $n>1$ case. It would be worthwhile studying this further.

\section{K3 Singularity and $c<1$ Topological String}

In section two, we have seen that topological string amplitudes on a Calabi-Yau manifold near the conifold are computable by transforming them into the $c=1$ string amplitudes. Here we will provide some evidence that topological string amplitudes on $K 3$ near the $A D E$ singularities are related to the $c<1$ topological strings [46], 477, 48] corresponding to the $A D E(1, n)$ matter coupled to gravity.

The first evidence for this relation is that the $y$-part of the superpotential (3.2) is that for the $N=2$ minimal model, whose topological twisting gives the standard description of the $c<1$ topological string [46]. The relation between the $c<1$ topological string and the 
twisted minimal model is further clarified in [49] where it is pointed out that the system of the Liouville field, the $(1, n)$ matter and the BRST ghosts has the $N=2$ superconformal symmetry of $\hat{c}=1-2 / n$ with the same chiral ring structure as that of the twisted $N=2$ minimal model of the $A_{n-1}$ type. However there is another choice of twist (by using the matter current instead of the Liouville) which leads to an $N=2$ algebra with $\hat{c}=1+2 / n$. This observation, in the case of $n=1$, led to the description of the $c=1$ string at the self-dual radius in terms of the Landau-Ginzburg model with $W=x^{-1}$ [15]. Therefore the $c<1$ string may also be related to the model with $W=x^{-n}$, in the case of the $A_{n-1}$ series. Curiously the superpotential (3.2) for the $A D E$ singularity is combining these two descriptions of the $c<1$ string.

The relation between the $W=x^{-n}$ potential and the $c<1$ string can be stated more explicitly. Let us first examine the case of $n=2$ by using the description in term of the Kazama-Suzuki model $S L(2, R)_{4} / U(1)$. In this case, the $U(1)$ current of the $N=2$ algebra (3.4) takes the form

$$
J=2 \psi \bar{\psi}-J^{3}
$$

and the BRST current is

$$
J_{B R S T}=C\left(\psi \bar{\psi}-J^{3}-i \partial X\right)
$$

In this case, the scalar $X$ lives on $S^{1}$ with $R=1$. We can then rewrite the $U(1)$ current $J$ as

$$
J=3 \psi \bar{\psi}-2 J^{3}-i \partial X-\left\{Q_{B R S T}, B\right\}
$$

After the topological twisting by $J,(\bar{\psi}, \psi)$ become of weights $(2,-1)$ and we may identify them as the BRST ghosts for diffeomorphism. Since $X$ is at radius $R=1$, we can fermionize it to $(b, c)$ which become of weights $(1,0)$ after the twisting. As in the case of the $c=1$ string, $(\beta, \gamma)$ of the Wakimoto construction of $S L(2, R)_{4}$ decouple together with $(B, C)$, and we are left with the scalar field $\phi$ with $c=27$. We can then combine $(b, c)$ and $\phi$ to make bosonic ghosts $(\tilde{\beta}, \tilde{\gamma})$ of weights $(2,-1)$. The resulting system of $(\bar{\psi}, \psi)$ and $(\tilde{\beta}, \tilde{\gamma})$ is nothing but the pure topological gravity [50], [51], [52].

For general $n$, the $U(1)$ current (3.4) of the Kazama-Suzuki model can be written as

$$
J=3 \partial \bar{\psi}-2 J^{3}-i \sqrt{\frac{2}{n}}(n-1) \partial X-2\left(1-\frac{1}{n}\right)\left\{Q_{B R S T}, B\right\}
$$


Therefore the topological twist by $J$ again transforms $(\psi, \bar{\psi})$ into the BRST ghosts of weights $(2,-1)$. The central charge for the scalar $\phi$ becomes

$$
c_{\phi}=1+6 \frac{(n+1)^{2}}{n}
$$

and the scalar $X$ has

$$
c_{X}=1-6 \frac{(n-1)^{2}}{n} .
$$

In other words the topological theory we are dealing is equivalent to the $(1, n)$ matter coupled to the two-dimensional gravity.

Thus we are led to conjecture that the topological string theory on the $A D E$ singularities (3.1) is related to the $c<1$ topological string [46], [47], 48]. In the case of $n>2$, however, there is a subtlety since we need to perform the orbifoldization of the LandauGinzburg model as in section two and therefore the $S L(2, R)_{n+2} / U(1)$ Kazama-Suzuki model and the $N=2$ minimal model are not completely decoupled. To clarify this point, let us work on the Landau-Ginzburg model directly.

For the $\hat{c}=2$ model, the most natural class of string amplitudes are $N=2$ string amplitudes [53], which can be expressed as $N=4$ topological string amplitudes [8]. Let us consider the $A_{n-1}$ singularity described by the superpotential

$$
W=-\mu x^{-n}+y^{n} .
$$

This potential can be deformed by adding chiral primary fields. In order to preserve the $N=4$ symmetry, we only allow chiral primary fields of charge 1 given by

$$
\phi_{i}=x^{i-n-1} y^{i-1} \quad(i=1,2, \ldots, n-1)
$$

In particular, $\phi_{i=1}$ changes the value of $\mu$. At genus- $0, N=4$ topological amplitudes are defined for four or higher points [8] as

$$
\begin{aligned}
A_{i_{1} \cdots i_{m}}^{0}=\int d^{2} z_{4} \cdots d^{2} z_{n}\langle & \phi_{i_{1}}(0) \phi_{i_{2}}(1) \phi_{i_{3}}(\infty) \times \\
& \left.\times J_{L}^{-} J_{R}^{-} \phi_{i_{4}}\left(z_{4}\right) G_{L}^{-} G_{R}^{-} \phi_{i_{5}}\left(z_{5}\right) \cdots G_{L}^{-} G_{R}^{-} \phi_{i_{n}}\left(z_{n}\right)\right\rangle,
\end{aligned}
$$

where $J^{-}$is one of the $S U(2)$ current of level 1 which belongs to the (small) $N=4$ algebra.

It turns out that the selection rule for this amplitude is consistent with that of the $(1, n+1)$ matter coupled to the two-dimensional gravity. The $(1, n+1)$ matter can be realized as a twisted $N=2$ minimal model [46] with the superpotential

$$
W(y)=y^{n+1} \text {. }
$$


This superpotential can be deformed by adding chiral primary fields given by

$$
\phi_{i}^{\prime}=y^{i} \quad(i=0,1, \ldots, n-1)
$$

If we consider four or higher points at genus-0, a topological string amplitude involving $\phi_{i=0}^{\prime}$ vanishes since $G^{-}$annihilates it. Therefore only $\phi_{i}^{\prime}$ with $(i=1, \ldots, n)$ are relevant in the comparison. By using the $U(1)$ charge conservation and the parafermion selection rule, it is straightforward to show that the selection rule for the $(1, n+1)$ topological string amplitude [46], [47], [48] satisfies the selection rule for the $N=4$ string amplitude (6.2) provided we identify $\phi_{i}^{\prime}$ with $\phi_{i}(i=1,2, \ldots, n-1)$. It is not yet clear why the shift $n \rightarrow n+1$ of the level takes place, and this merits further investigation.

We would like to thank S. Shenker, who participated in an earlier stage of this project, for discussions. We also thank the Aspen Center for Physics, where this project was initiated. The work of HO was supported in part by the National Science Foundation under grant PHY-9501018 and in part by the Director, Office of Energy Research, Office of High Energy and Nuclear Physics, Division of High Energy Physics of the U.S. Department of Energy under Contract DE-AC03-76SF00098. The research of CV was supported in part by NSF grant PHY-92-18167. 


\section{References}

[1] C.M. Hull and P.K. Townsend, "Unity of superstring dualities," Nucl. Phys. B438 (1995) 109.

[2] E. Witten, "String theory dynamics in various dimensions," Nucl. Phys. B443 (1995) 85.

[3] A. Strominger, "Massless black holes and conifolds in string theory", Nucl. Phys. B451 (1995) 96.

[4] J. Polchinski, "Dirichlet branes and Ramond-Ramond charges," e-Print Archive: hepth/9510017.

[5] M. Bershadsky, C. Vafa and V. Sadov, "D-strings and D-manifolds," e-Print Archive: hep-th/9510225.

[6] B.R. Greene, A. Shapere, C. Vafa and S.-T. Yau, "Stringy cosmic strings and noncompact Calabi-Yau manifolds," Nucl. Phys. B337 (1990) 1.

[7] D. Ghoshal and C. Vafa, " $c=1$ string as the topological theory of the conifold," e-Print Archive: hep-th/9506122.

[8] N. Berkovits and C. Vafa, "N=4 Topological Strings," Nucl. Phys. B433 (1995) 123.

[9] M. Bershadsky, S. Cecotti, H. Ooguri and C. Vafa, "Holomorphic Anomalies in Topological Field Theories," Nucl. Phys. B405 (1993) 279.

[10] M. Bershadsky, S. Cecotti, H. Ooguri and C. Vafa, "Kodaira-Spencer theory of gravity and exact results for quantum string amplitudes", Commun. Math. Phys. 165 (1994) 311.

[11] I. Antoniadis, E. Gava, K.S. Narain and T.R. Taylor, "Topological amplitudes in string theory," Nucl. Phys. B413 (1994) 162.

[12] P. Candelas, A.M. Dale, C.A. Lütken, and R. Schimmrigk, "Complete Intersection Calabi-Yau Manifolds", Nucl. Phys. B298 (1988) 493; P.S. Green and T. Hübsch, "Possible Phase Transitions Among Calabi-Yau Compactifications", Phys. Rev. Lett. 61 (1988) 1163; "Connecting Moduli Spaces of Calabi-Yau Threefolds", Commun. Math. Phys. 119 (1988) 431; P. Candelas, P.S. Green, and T. Hübsch, "Finite Distance Between Distinct Calabi-Yau Manifolds", Phys. Rev. Lett. 62 (1989) 1956; "Rolling Among Calabi-Yau Vacua", Nucl. Phys. B330 (1990) 49; P. Candelas and X.C. de la Ossa, "Comments on Conifolds", Nucl. Phys. B342 (1990) 246.

[13] P. Candelas, A. Font, S. Katz and D. Morrison, "Mirror symmetry for two parameter models 1" Nucl. Phys. B416 (1994) 481; "2," Nucl. Phys. B429 (1994) 626; S. Hosono, A. Klemm, S. Theisen and S.-T. Yau, "Mirror symmetry, mirror map and applications to complete intersection Calabi-Yau spaces," Nucl. Phys. B433 (1995) 501.

[14] C. Vafa, "A stringy test of the fate of the conifold", Nucl. Phys. B447 (1995) 252.

[15] S. Mukhi and C. Vafa, "Two-dimensional black-hole as a topological coset model of $c=1$ string theory," Nucl. Phys B371 (1992) 191. 
[16] Y. Kazama and H. Suzuki, "New $N=2$ superconformal field theories and superstring compactification," Nucl. Phys. B321 (1989) 232.

[17] E. Witten, "The N matrix model and gauged WZW models", Nucl. Phys.B371 (1992) 191.

[18] I. Klebanov and D. Gross, " One-dimensional string theory on a circle", Nucl. Phys. B344 (1990) 475.

[19] J. Distler and C. Vafa, "A critical matrix model at $c=1$ ", Mod. Phys. Lett. A6 (1991) 259.

[20] E.J. Martinec, "Algebraic geometry and effective Lagrangians," Phys. Lett. 217B (1989) 431.

[21] C. Vafa and N. Warner, "Catastrophes and the classification of conformal theories," Phys. Lett. 218B (1989) 51.

[22] D. Ghoshal and S. Mukhi, "Topological Landau-Ginzburg model for two-dimensional string theory", Nucl. Phys.B425 (1994) 173.

[23] A. Hanany, Y. Oz and R. Plesser, "Topological Landau-Ginzburg formulation and integrable structure of 2-D string theory",Nucl. Phys. B425 (1994) 150.

[24] D. Ghoshal and S. Mukhi, "Landau-Ginzburg model for a critical topological string," to appear in the Proceedings of the International Colloquium of Modern Quantum Field Theory, eds S. Das et al.

[25] B.R. Greene, C. Vafa and N.P. Warner, "Calabi-Yau manifolds and renormalization group flow," Nucl. Phys. B324 (1989) 371.

[26] E. Witten, "Phases of $N=2$ theories in two dimensions", Nucl. Phys.B403 (1993) 159.

[27] I. Antoniadis, E. Gava, K.S. Narain and T.R. Taylor, " $N=2$ type II - heterotic duality and higher derivative F-terms," e-Print Archive: hep-th/9507115.

[28] E. Witten, "On string theory and black holes," Phys. Rev. D44 (1991) 314.

[29] R. Dijkgraaf, H. Verlinde and E. Verlinde, " String propagation in a black hole geometry," Nucl. Phys. B371 (1992) 269.

[30] L.J. Dixon, M.E. Peskin and J. Lykken, " $N=2$ superconformal symmetry and SO $(2,1)$ current algebra, Nucl. Phys. B325 (1989) 329.

[31] E. Witten, "Some comments on string dynamics," e-Print Archive: hep-th/9507121.

[32] J. Polchinski and E. Witten, "Evidence for heterotic - type I string duality," e-Print Archive: hep-th/9510169.

[33] P.S. Aspinwall, "Enhanced gauge symmetries and $K 3$ surfaces," Phys. Lett. 357B (1995) 329.

[34] D. Gepner, "Exactly solvable string compactification on manifolds of $S U(N)$ holonomy," Phys. Lett. 199B (1987) 380.

[35] C.G. Callan, J.A. Harvey and A. Strominger, "Worldsheet approach to heterotic solitons and instantons," Nucl. Phys. B359 (1991) 611. 
[36] F. Ravanini and S.-K. Yang, "Modular invariance in $N=2$ superconformal field theories," Phys. Lett. 195B (1987) 202.

[37] A. Cappelli, C. Itzykson and J.B. Zuber, "The $A D E$ classification of minimal and $A_{1}^{1}$ conformal invariant theories," Commun. Math. Phys. 113 (1987) 1.

[38] T. Banks and L. Dixon, "Constraints on string vacua with space-time supersymmetry," Nucl. Phys. B307(1988) 93.

[39] T. Eguchi, H. Ooguri, A. Taormina and S.-K. Yang, "Superconformal algebras and string compactification on manifolds with $S U(N)$ holonomy, Nucl. Phys. B315(1989) 193.

[40] A. Sevrin, W. Troost and A. Van Proeyen, "Superconformal algebras in two dimensions with $N=4$," Phys. Lett. 208B 1988.

[41] C. Vafa, "Quantum symmetries of string vacua," Mod. Phys. Lett. A4 (1989) 1615.

[42] G. Horowitz and A. Strominger, "Black strings and p-branes," Nucl. Phys. B360 (1991) 197.

[43] C.G. Callan, J.A. Harvey and A. Strominger, "Supersymmetric string solitons," in Proceeding of the 1991 Trieste Spring School and String Theory and Quantum Gravity, eds.

[44] T. Hübsch, "Calabi-Yau manifolds, a bestiary for physicists," (World Scientific, 1992).

[45] D. Ghoshal, P. Lakdawala and S. Mukhi, Mod. Phys. Lett. A8 (1993) 3187.

[46] K. Li, "Topological gravity with minimal matter," Nucl. Phys. B354 (1991) 711; "Recursion relations in topological gravity with minimal matter," Nucl. Phys. B354 725.

[47] R. Dijkgraaf and E. Witten, "Mean field theory, topological field theory, and multimatrix models," Nucl. Phys. B342 (1990) 486.

[48] R. Dijkgraaf, E. Verlinde and H. Verlinde, "Topological strings in $d<1$, Nucl. Phys. B352 (1991) 59.

[49] B. Gato-Riviera and A.M. Semikhatov, "Minimal models from $W$ constrained hierarchies via the Kontsevich-Miwa transform," Phys. Lett. B288 (1992) 38; M. Bershadsky, W. Lerche, D. Nemeshansky and N.P. Warner, "Extended $N=2$ superconformal structure of gravity and W gravity coupled to matter," Nucl. Phys. B401 (1993) 304.

[50] E. Witten, "On the structure of the topological phase of two-dimensional gravity," Nucl. Phys. B340 281.

[51] J. Distler, "2-d quantum gravity, topological field theory and multicritical matrix models," Nucl. Phys. B342 523.

[52] E. Verlinde and H. Verlinde, "A solution of two-dimensional topological quantum gravity," Nucl. Phys. B348 457.

[53] H. Ooguri and C. Vafa, "Selfduality and $N=2$ String Magic," Mod. Phys. Lett. A5 (1990) 1389; H. Ooguri and C. Vafa, "Geometry of $N=2$ Strings," Nucl. Phys. B361 (1991) 469 . 